\title{
COMPARATIVE ANALYSIS OF POTENTIALITY OF ESCULIN AND HINOKITOL (B-THUJAPLICIN) AS ANTI-PARKINSONISM DRUGS: A PILOT IN SILICO STUDY
}

\author{
KRISHNAPRIYA MADHU VARIERa, SUMATHI THANGARAJAN ${ }^{*}$, ARULVASU CHINNASAMYb, \\ GOPALSAMY BALAKRISHNANc, RENJITH PAULOSEc
}

\begin{abstract}
aDepartment of Medical Biochemistry, Dr. A. L. M. Post Graduate Institute of Basic Medical Sciences, University of Madras, Taramani Campus, Chennai, India, bepartment of Zoology, University of Madras, Guindy Campus, Chennai, India, cLiatris Biosciences LLP, Cochin, India Email: drsumathi.bioscience@gmail.com
\end{abstract}

Received: 23 Sep 2016 Revised and Accepted: 08 Nov 2016

\section{ABSTRACT}

Objective: Parkinson's disease (PD) is a leading cause of mental disability and death worldwide. Even though there are many advances in drug development against PD, a potent low dosage drug with fewer side effects are still in its nursery. This is a pioneer in silico attempt to test the anti-PD actions of esculin and hinokitol to act novel drugs.

Methods: In this study, using Auto dock tools 4.2, esculin and hinokitol ( $\beta$-Thujaplicin) were predicted for its inhibitory actions with Alpha-Synuclein (AS) Apo site, Dopamine D3 Receptor (D3R), Glycogen Synthase Kinase-3 Beta (GSK3ß), Mono Oxidase B (MAO-B), Parkin and Tyrosine 3-Hydroxylase $(\mathrm{TH})$ with levodopa standard. The reliability of the 3D predicted model of these proteins were analysed using RAMPAGE. Further, the blood-brain barrier (BBB) crossing ability of the natural compounds were analysed using cbligand. The In silico ADME (Absorption, Distribution, Metabolism, Excretion) properties of esculin and hinokitol were compared with that of levodopa using molinspiration and admetSAR @ LMMD software.

Results: The predictions were that hinokitol, being blood-brain barrier positive (BBB+) with fewer side effects could be a potent anti-PD drug than esculin as it proved to be blood-brain barrier negative (BBB-). Hinokitol was predicted to be good inhibitors of AS, MAO-B and Parkin.

Conclusion: The study revealed that hinokitol could be a potent anti-PD drug, being BBB+. Hinokitol was additionally predicted as a good inhibitor of AS, MAO-B and Parkin than levodopa standard.

Keywords: AutoDock 4.2, Esculin, Hinokitol, Rampage, Admet SAR, Molinspiration

(C) 2017 The Authors. Published by Innovare Academic Sciences Pvt Ltd. This is an open access article under the CC BY license (http://creativecommons.org/licenses/by/4. 0/) DOI: http://dx.doi.org/10.22159/ijpps.2017v9i1.15340

\section{INTRODUCTION}

Among the entire world population, PD, is the second most common neurodegenerative disease affecting ageing populations with a prevalence of $0.3 \%$ [1]. PD, is one of the prominent, progressive movement disorder characterised by postural instability, rigidity, resting tremor and bradykinesia [1], resulting essentially from neurolysis affecting dopaminergic neurones in the substantia nigra pars compacta (SNpc). Furthermore, supposing the role of several neurotransmitters in PD, several neuro-structures, such as anterior olfactory structures, amygdala, dorsal motor nucleus of the vagus, caudal raphe nuclei, locus coeruleus, autonomic nervous system, hippocampus, and cerebral cortex has been linked to PD. In addition, non-motor symptoms, including anxiety and depression, olfactory and memory impairments, sleep abnormalities, as well as gastrointestinal disturbance are also prevalent in PD patients [2].

PD, multiple system atrophy and dementia show expression of alpha synuclien (AS) with prominent lewy bodies [3]. AS, which are the histological hallmarks of PD is the major constituent of proteinaceous amyloidogenic inclusions known as lewy bodies, among PD patients. The $14 \mathrm{kDa}$ polypeptide AS, along with the death of dopaminergic neurones within the SN of the brain, makes the disease expressed prominently. In an in vitro isolated system, AS accumulates instead of growing into mature fibrils as 5-hydroxytryptamine receptors (5-HT) binds and stabilises the aggregates [2]. GSK3 $\beta$, one of the GSK3 isomers, an appealing therapeutic target for neurodegenerative disorders plays a major role in neuronal apoptosis. Moreover, its inhibition decreases expression of AS, which make this kinase much more attractive for neurobiologists [4].

Catechol-O-methyl transferase (COMT) and monoamine oxidase (MAO) inhibitors will prolong the half-life of levodopa and dopamine [5-7]. An altered MAO-B activity has been observed in PD patient's platelets, which was implicated in the PD neuropathology for its role in metabolizing the neurotoxin 1-methyl-4-phenyl-1,2,3,6- tetra-hydro pyridine, and forming $\mathrm{H}_{2} \mathrm{O}_{2}$ during dopamine metabolism [8].

In the prefrontal cortex, inhibition of D3R enhances dopamine and acetylcholine release. In addition, D3R, modulates the prefrontal cortex cholinergic levels [9]. Decarboxylation of L-3,4-dihydroxyphenylalanine (L-DOPA) and L-5-hydroxytryptophan by DOPA decarboxylase (DDC) is responsible for the synthesis of the key neurotransmitters dopamine and serotonin respectively. DDC is one of the major markers of PD and hypertension [10]. Most Parkin knockout mouse models failed to develop the pathophysiology of PD and display the selective dopamine (DA) neurone loss. This new understanding of the mechanism and the importance of mitochondrial quality control may also extend to other common neurodegenerative diseases associated with mitochondrial dysfunction such as Alzheimer's disease and Amyotrophic lateral sclerosis [11]. It has been documented that TH phosphorylation during ageing decreases in the striatum, which was the indication of striatal DA loss in human. Thus, the striatal TH regulation during ageing by $>70 \%$ loss of striatal DA will result in the appearance of loco motor symptoms associated with PD [12].

Recently, bioactive compounds, have been isolated from various plant materials, with potential antineuralgic effects. These bioactive compounds mainly include plant secondary metabolites like saponins, steroids, flavanones, quinones, glycosides and so on. Esculin is a glucoside coumarin isolated from Aesculus hippocastanum (Bark), Artemisia vulgaris, Cichorumintybus (Leaf and Flower), Crataeguslavigata (Bark, Leaf and fruit), and Crataegusmonogyna (Bark), famous for its anti-inflammatory, anti-helminthic, anti-microbial, gastroprotective, anti-cancer as well as anti-oxidant properties. Moreover, this multipotent tricyclic coumarin is reported well as promising leads for developing anti-Alzheimer agents [13]. The anti-oxidant property of esculin is further elucidated through a reduction in GSH and SOD levels [14]. Hinokitiol, a tropolone-related compound found in the heartwood 
cupressaceous plants, is widely used in hair tonics, toothpaste, cosmetics, and food as an antimicrobial agent. It is reported as an antioxidant agent, anticancer agent, and a potent inhibitor of mushroom tyrosinase. Tropolones are reported as powerful cholesterinase inhibitors [15]. Furthermore, hinokitol, is reported for its neurodefensive action against ischemic brain injury induced in rats [16]. In this paper, as a pioneer in silico study, the bio activeness of plant secondary metabolites, esculin and hinokitol were compared by predicting their neuroprotective action by inhibiting AS, D3R, GSK3 $\beta$, MAO-B, Parkin and TH receptors involved in PD neuropathology keeping levodopa as standard.

\section{MATERIALS AND METHODS}

\section{Software's}

The receptor model was built by using Auto Dock tools 1.5.6 and MGL tools 1.5.6 packages (The Scripps Research Institute, Molecular Graphics Laboratory, and 10550 North Torrey Pines Road, CA, 92037). Cbligand tools were used to check for in silico BBB crossing ability of the ligands. Molinspiration tools were used for analysing ADME and molecular physio-chemical properties of levodopa, esculin and hinokitol. Toxicity prediction was done by admetSAR @ LMMD. The reliability of the 3D predicted models of the protein and receptor models were analyzed using RAMPAGE.

\section{Molecular docking studies}

The 3-dimensional structure of Levadopa (PubChem ID: 6047), Esculin (PubChem ID: 161211025) and Hinokitol (PubChem ID: 3611) were retrieved from PubChem and optimised for docking using Discovery studio. The protein Crystallographic structures of receptors Alpha-Synuclein Apo site (3Q25), Dopamine D3 Receptor (3BPL), Glycogen Synthase Kinase-3 Beta (1H8F), Mono Oxidase B (10JA), Parkin (5C9V) and Tyrosine 3-Hydroxylase (1TOH) were retrieved from www. rcsb. Org was prepared for docking by deleting all heteroatoms, ligands and water molecules and optimised by minimization of energy by using Discovery studio. The obtained structures were saved and used for the docking studies. The 3D structure of proteins was generated after optimisation in Discovery studio. Auto Dock tools automatically compute gasteiger charges and determine rotatable bonds of the ligand to be able to generate different conformers for the docking [17]. Receptor grids were generated using $40 \times 40 \times 40$ grid points in xyz with a grid spacing of $1.000 \AA$ A. Grid boxes were generated using autogrid4. The lamarckian genetic algorithm was used for all molecular docking simulations. The interactions of the protein-ligand complexes were also retrieved through Auto Dock tools 1.5.6. Dockings were carried out with following parameters for the number of runs: 50, population size: 150, the number of evaluations: $2,500,000$ and number of generations: 27,000 numbers of generations $[18,19]$.

\section{Ramachandran plot rampage analysis}

Ramachandran plot was used to check the reliability of the predicted 3D model. The prepared structures of all the six receptors were checked for the structural reliability through rampage analysis [20].

\section{ADME studies}

An in silico study of the synthesised compounds was performed for the prediction of ADME properties. Total polar surface area (TPSA), miLog $\mathrm{P}$, the number of rotatable bonds, the number of a hydrogen donor and acceptor atoms were calculated upon the basis of Lipinski's rule of five [21]. BBB prediction was done by utilising a web-based program (www. cbligand. org/BBB). The compounds with positive values can cross readily in the BBB, while compounds with negative values are poorly distributed across the brain.

\section{Toxicity estimation}

It was noticed that too many compounds were terminated in clinical development because of unsatisfactory pharmacokinetics or in silico ADME studies. It is now possible to predict the activity and toxicity risks of compounds through reliable Bioinformatics tools, e. g., admetSAR @ LMMD. In the present study, we have calculated toxicity risk parameters such as mutagenicity, tumorigenicity, and biodegradability of selected drugs; esculin and hinokitol with the standard drug levodopa.

\section{RESULTS}

\section{AutoDock}

AutoDock is performed by evaluating energies for both the bound and unbound states. It also incorporates a new charge-based desolvation method that uses a typical set of atom types and charges. The binding energy of the drugs; namely esculin and hinokitol were compared with levodopa, which showed the binding energies varying from-5.3 to-9.1 Kcal/Mol. The overall inhibitory action of esculin and hinokitol with the selected receptors in table 1 has to be appreciated with regard to standard levodopa. Alpha synuclien Apo site happened to be a potent prospective site of inhibitory action for esculin (-9.1 Kcal/Mol) than hinokitol (-6.9 $\mathrm{Kcal} / \mathrm{Mol})$, when compared with standard levodopa (-7.3 Kcal/Mol). The GSK3 $\beta$ showed-7.2 Kcal/mol of inhibitory energy with esculin. While it was only-5.6 Kcal/Mol for hinokitol and much lesser than levodopa, which was-5.8 Kcal/Mol. MOA-B is another receptor, which was highly inhibited by esculin with a prognostic favourable energy of-9.6 Kcal/Mol. Parkin revealed its maximum inhibitory action with esculin, being-7.4Kcal/Mol. The TH showed a pretty considerable amount of inhibition with the selected drugs likely as$8.8 \mathrm{Kcal} / \mathrm{Mol}$ for esculin and-7.1Kcal/Mol against hinokitol respectively. The possible active site amino acids, which are interacting with the sites, are indicated in fig. 1-6.

Table 1: Auto Dock Vina 4.2 results showing binding energy/affinity energy (Kcal/Mol) of receptors with the ligands

\begin{tabular}{|c|c|c|c|}
\hline \multirow[t]{2}{*}{ Receptors (PDB ID) } & \multicolumn{3}{|c|}{ Ligand (PUBCHEM ID) } \\
\hline & Levodopa (6047) & Esculin (161211025) & Hinokitol/ $\beta$-Thujaplicin (3611) \\
\hline$\alpha$-Synuclein Apo site (3Q25) & -7.3 & -9.1 & -6.9 \\
\hline Dopamine D3 Receptor (3BPL) & -5.8 & -8.3 & -6.3 \\
\hline Glycogen Synthase Kinase- $3 \beta(1 \mathrm{H} 8 \mathrm{~F})$ & -5.8 & -7.2 & -5.6 \\
\hline Mono Oxidase B (10JA) & -7.7 & -9.6 & -7.2 \\
\hline Parkin $(5 \mathrm{C} 9 \mathrm{~V})$ & -5.3 & -7.4 & -5.1 \\
\hline Tyrosine 3-Hydroxylase (1TOH) & -6.9 & -8.8 & -7.1 \\
\hline
\end{tabular}
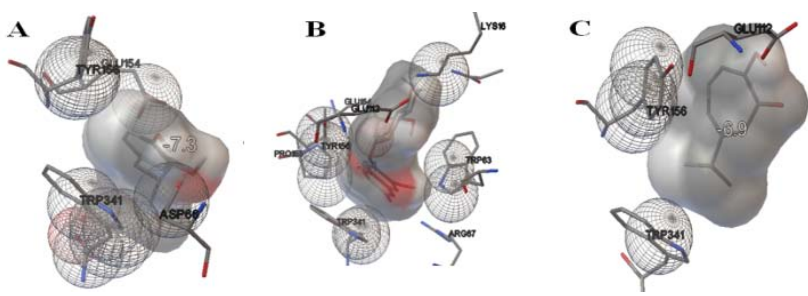

Fig. 1: The fig. showing active site interactions between Alpha-synuclein Apo site and ligands. A. Between alpha-synuclein Apo site and levodopa B. between alpha-synuclein Apo site and esculin C. between alpha-synuclein Apo site and hinokitol 

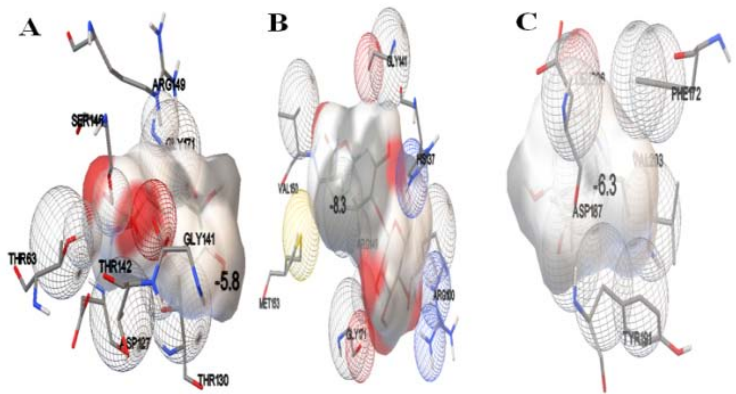

Fig. 2: The fig. showing active site interactions between dopamine D3 Receptors and ligands. A. Between dopamine D3 Receptors and levodopa B. Between dopamine D3 Receptors and esculin C. Between dopamine D3 Receptors and hinokitol
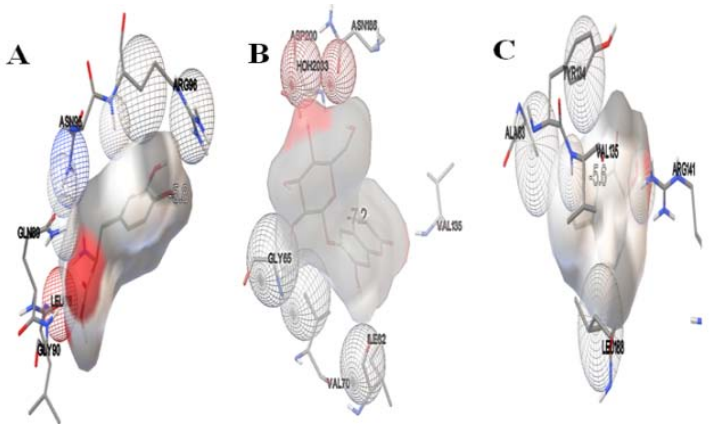

Fig. 3: The fig. showing active site interactions between glycogen synthase kinase-3-beta and ligands. A. between glycogen synthase kinase-3-beta and levodopa $B$. between glycogen synthase kinase-3-beta and esculin $C$. between

glycogen synthase kinase-3-beta and hinokitol
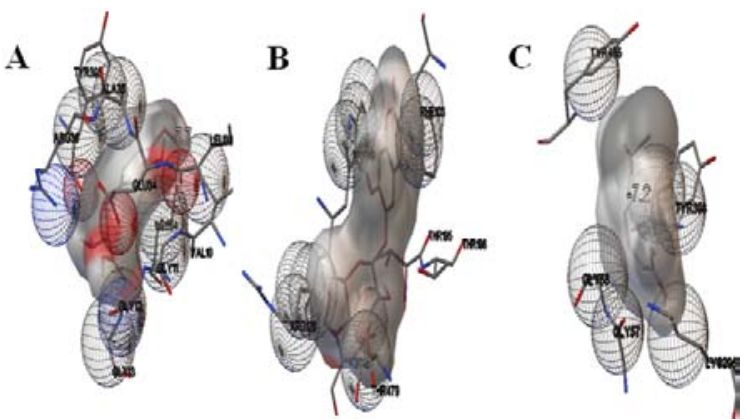

Fig. 4: The fig. showing active site interactions between mono oxidase $B$ and ligands. A. between mono oxidase $B$ and levodopa

B. Between mono oxidase B and esculin C. Between mono oxidase $B$ and hinokitol
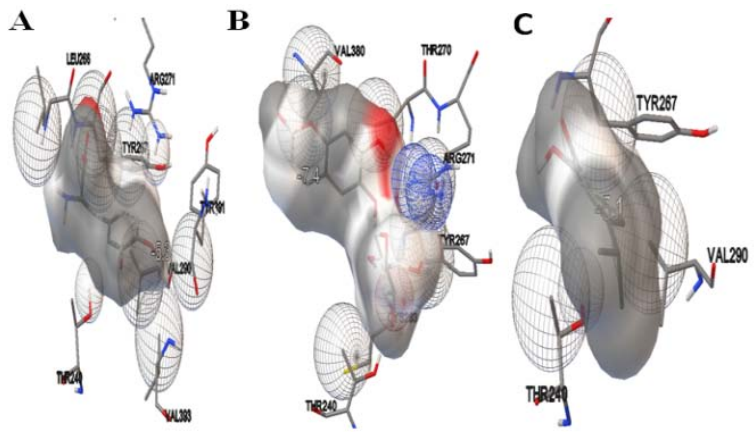

Fig. 5: The fig. showing active site interactions between Parkin and ligands. A. Between parkin and levodopa B. between parkin and esculin C. Between parkin and hinokitol
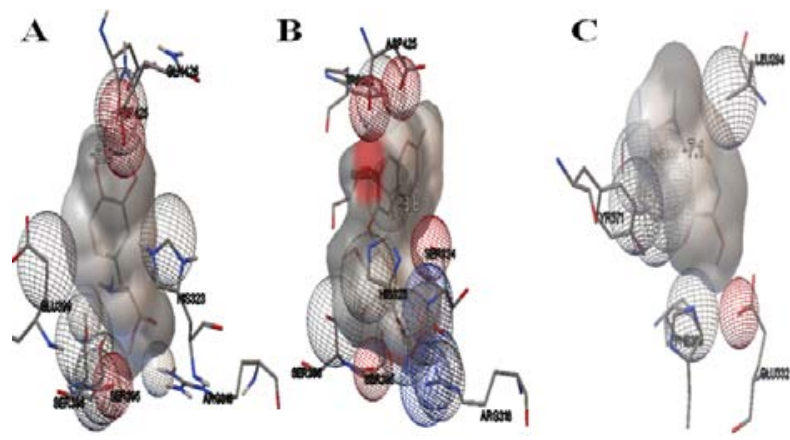

Fig. 6: The fig. showing active site interactions between tyrosine 3-hydroxylase and ligands. A. between tyrosine 3-hydroxylase and levodopa B. Between tyrosine 3-hydroxylase and esculin C. between tyrosine 3-hydroxylase and hinokitol

\section{Rampage analysis}

The percentage of residues in the favoured region for all the selected six receptors varied from 91.1-98.0. The same for MOA-B was found to be $98 \%$. The receptors like D3R and Parkin offered $96 \%$ of the favoured region amino acid configuration. Around $95 \%$ for synuclien were appraised for the favourable residues, which could be assessed under the active sites on the receptors. Meanwhile, it was $91 \%$ for GSK3beta and $90 \%$ in the MOA-B (table 2, fig. 7-12).

\section{Blood-brain barrier permeability}

According to the program www. cbligand. Org/BBB, the blood-brain barrier permeability of the selected two drugs along with standard levodopa was assessed. This indicates that the drug esculin was unable to cross the BBB (fig. 13-15). With regard to hinokitol and levodopa, both were BBB permeable with a higher permeability rate with levodopa (permeability score: +0.681) than hinokitol (Permeability Score: 0.317 ). While esculin with permeability score0.043 was found to be BBB-(table 3)
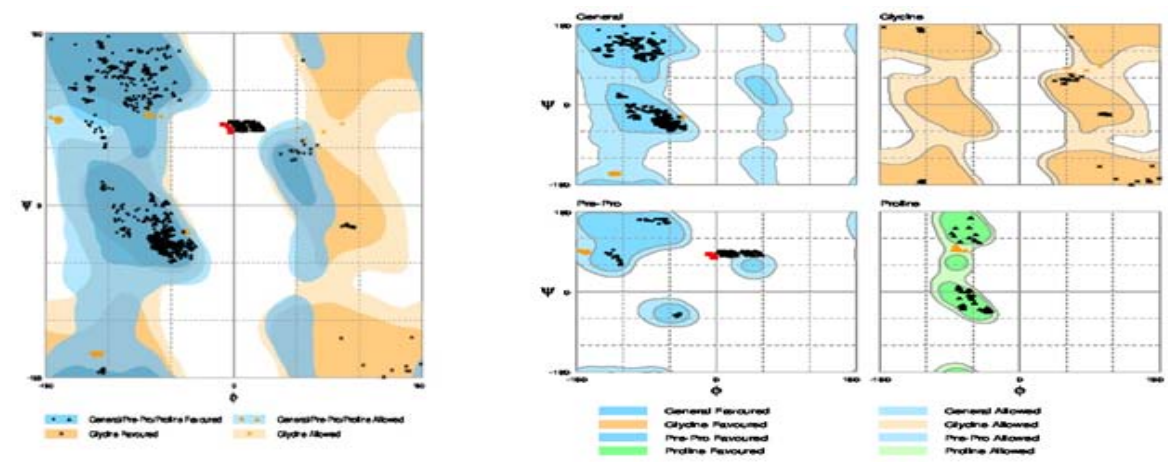

Fig. 7: Ramachandran plot analysis of alpha-synuclein Apo site 

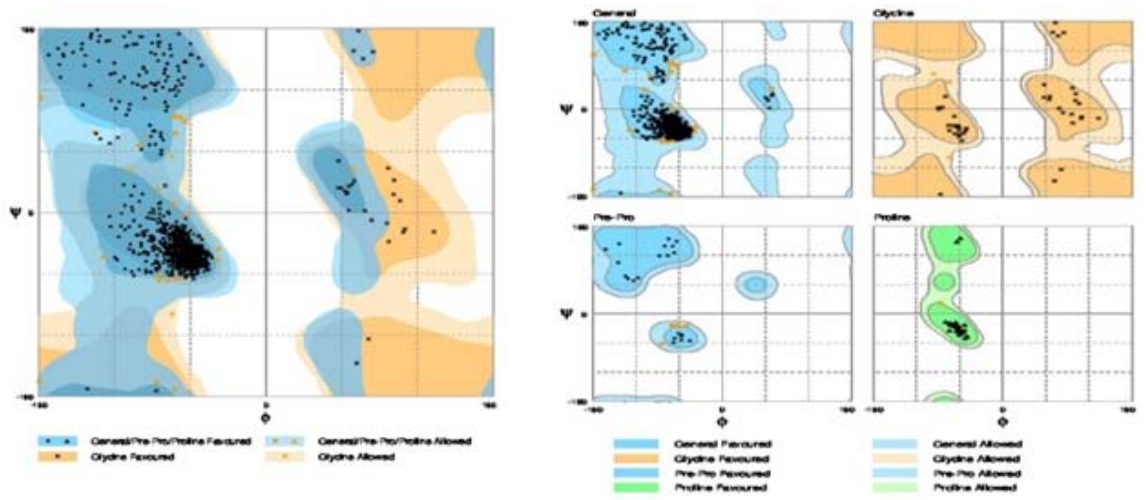

Fig. 8: Ramachandran plot analysis of human dopamine 3 receptors
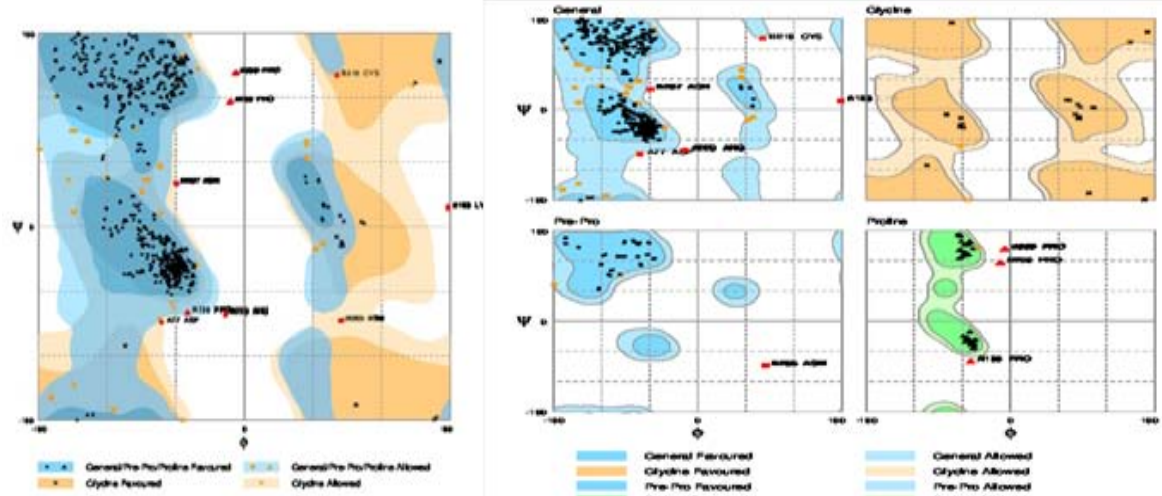

Fig. 9: Ramachandran plot analysis of human glycogen kinase $3 \beta$
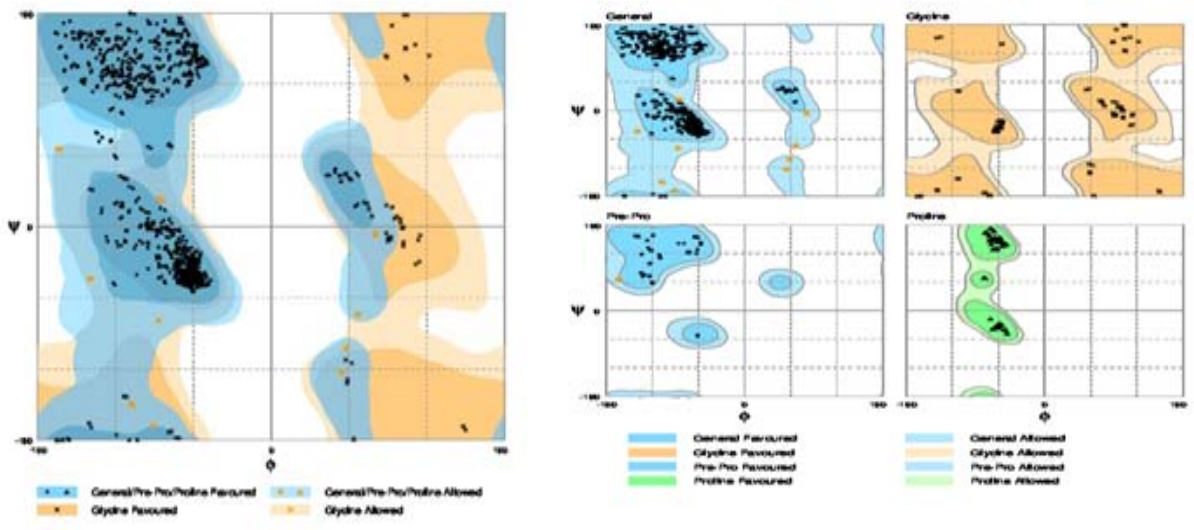

Fig. 10: Ramachandran plot Analysis of human Mono Oxidase B
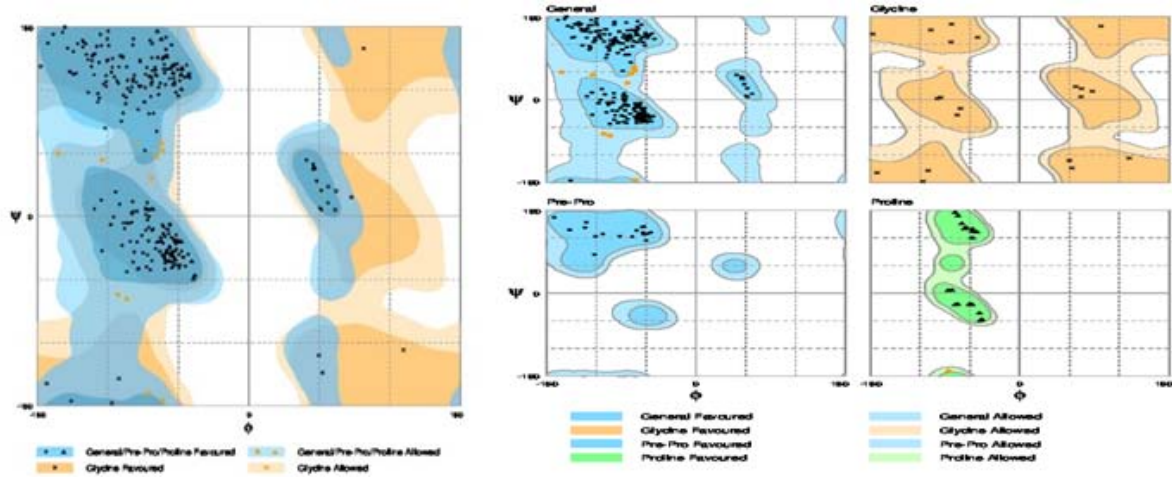

Fig. 11: Ramachandran plot Analysis of parkin 

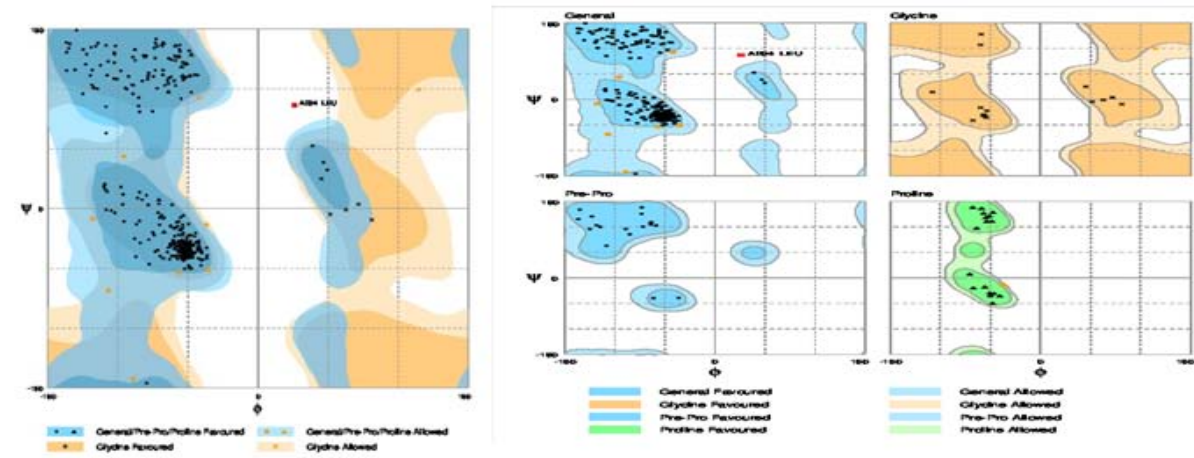

Fig. 12: Ramachandran plot analysis of human tyrosine 3-hydroxylase

Table 2: The rampage stereochemical quality of receptors by analyzing residue geometry and overall structure geometry

\begin{tabular}{|c|c|c|c|c|c|}
\hline \multirow[t]{2}{*}{ Receptor(PDB ID) } & \multicolumn{2}{|c|}{ Expected percentage(\%)of residues in } & \multicolumn{3}{|c|}{ Actual percentage (\%) of residues in } \\
\hline & Favored region & Allowed region & Favored region & Allowed region & Outlier region \\
\hline$\alpha$-Synuclein Apo site (3Q25) & 98.00 & 2.00 & 95.40 & 3.70 & 0.9 \\
\hline Dopamine D3 Receptor (3BPL) & 98.00 & 2.00 & 96.2 & 3.8 & 0.0 \\
\hline Glycogen Synthase Kinase- $3 \beta(1 \mathrm{H} 8 \mathrm{~F})$ & 98.00 & 2.00 & 91.1 & 6.6 & 2.3 \\
\hline Mono Oxidase B (10JA) & 98.00 & 2.00 & 98.0 & 2.0 & 0.0 \\
\hline Parkin $(5 \mathrm{C} 9 \mathrm{~V})$ & 98.00 & 2.00 & 96.3 & 3.7 & 0.0 \\
\hline Tyrosine 3-Hydroxylase (1TOH) & 98.00 & 2.00 & 96.9 & 2.8 & 0.3 \\
\hline
\end{tabular}
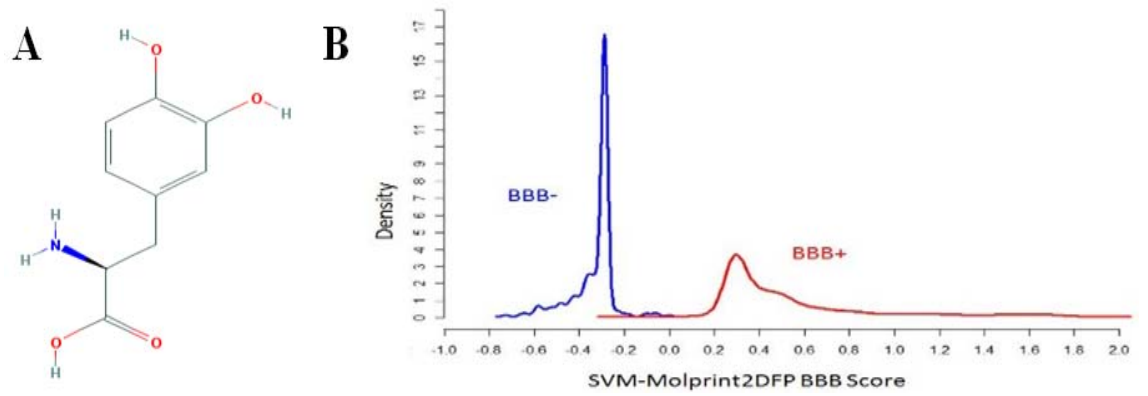

Fig. 13: Blood brain barrier permeability scores for levodopa. A. Pub Chem 2D structure of levodopa. B. Graph showing BBB permeability score of levodopa
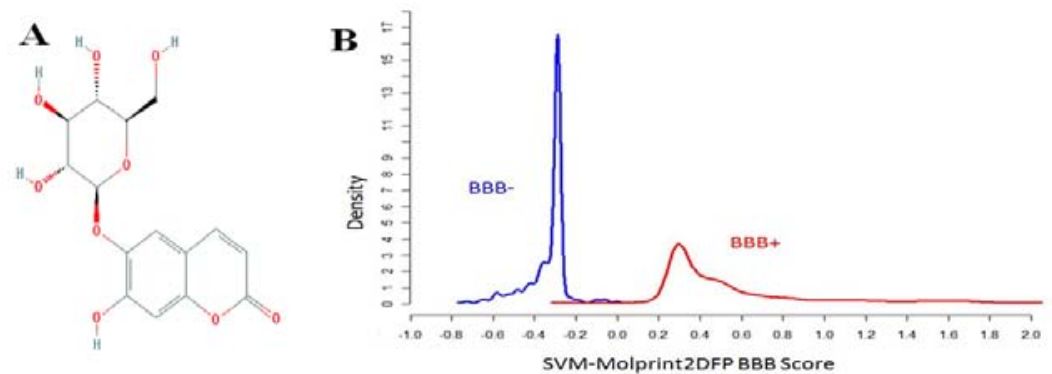

Fig. 14: Blood-brain barrier permeability scores for esculin A. PubChem 2D structure of esculin B. graph showing BBB permeability score of esculin
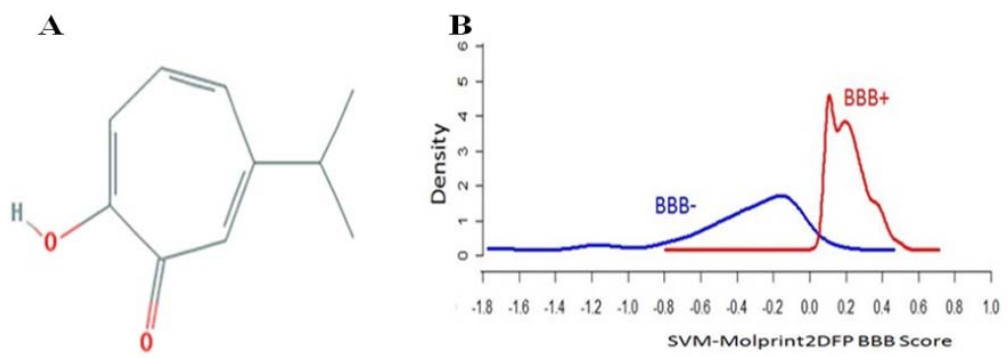

Fig. 15: Blood-brain barrier permeability scores for hinokitol A. Pub Chem 2D structure of hinokitol B. graph showing BBB permeability score of hinokitol 


\section{Molecular physio-chemical properties}

The in silico studies suggested that the Total Polar Surface Area (TPSA) for hinokitol was 37.30 and for esculin was 149.82. While TPSA for levodopa was 103. 78. The mi log P for hinokitol and esculin was +2.08 and-0.99 respectively. While the same for levodopa was-2.20 (table 3).

\section{Bioactivity studies}

The bioactivity studies revealed the possible bioactivity scores of the drugs to act as a potent G-protein coupled receptor (GPCR) ligand, ion channel modulator, kinase modulator, nuclear receptor ligand, protease inhibitor as well as an enzyme inhibitor (table 4).

The study exposed the bioactivity scores of hinokitol as a strong GPCR ligand with-1.19 score. Hinokitol can be further treated as a potential nuclear receptor ligand (bioactivity score:-1.02) and a protease inhibitor (bioactivity score:-1.27).

\begin{abstract}
ADMET studies
The ADMET (Absorption, Distribution, Metabolism, Excretion and Toxicity) studies, showed the selected drugs can easily undergo human intestinal digestion and are non-inhibitors of P-glycoproteins (table 5). They even act as non-inhibitor of renal organic cations. With regard to distribution and metabolism, compared to standard levodopa, the properties such as CYP450 2C9 substrate, CYP450 2D6 substrate, CYP450 3A4 substrate CYP450 1A2 inhibitor CYP450 2C9 inhibitor CYP450 2D6 inhibitor CYP450 2C19 inhibitor CYP450 3A4 inhibitor and CYP inhibitory promiscuity were exactly same for esculin and standard Levodopa. When the excretion and toxicity mechanism of the drugs are concerned, all the three drugs are non-inhibitor of human etheragogo related gene inhibition and are non-carcinogens. Unlike hinokitol, esculin was AMES negative, showing its non-mutagenicity. The regression profile estimated the rat acute toxicity (LD50, Mol/kg) for esculin and hinokitol as 2.2896 and 2.7972 than levodopa being 2.3137 (LD50, $\mathrm{Mol} / \mathrm{kg}$ ). The fish toxicity (pLC50, mg/l) was 1.0043, 1.3261 and-1.4952 for levodopa, esculin and hinokitol respectively.
\end{abstract}

Table 3: Calculation of molecular physiochemical properties

\begin{tabular}{lllllllll}
\hline Compound & $\begin{array}{l}\text { Number } \\
\text { of atoms }\end{array}$ & Molecular weight & TPSA & miLogP & $\begin{array}{l}\text { N } \\
\text { Rtb }\end{array}$ & nON & $\begin{array}{l}\text { nOHNH } \\
\text { Rule of } \\
\text { Violation }\end{array}$ & $\begin{array}{l}\text { BBB Permeability scores } \\
\text { (+/-) }\end{array}$ \\
\hline Levodopa & 14 & 197.19 & 103.78 & -2.20 & 3 & 5 & 5 & 0 \\
Esculin & 24 & 340.28 & 149.82 & -0.99 & 3 & 9 & 5 & 0 \\
Hinokitol & 12 & 164.20 & 37.30 & +2.08 & 1 & 2 & 1 & -0.681 \\
\hline
\end{tabular}

n=number, TPSA=Total Polar Surface Area, Log P=octoanol/water coefficient

Table 4: Bioactivity predictions using molinspiration tools

\begin{tabular}{|c|c|c|c|}
\hline \multirow[t]{2}{*}{ Bioactive properties } & \multicolumn{3}{|c|}{ Bioactivity scores } \\
\hline & Levodopa & Esculin & Hinokitol \\
\hline GPCR Ligand & -0.04 & -0.10 & -1.19 \\
\hline Ion Channel Modulator & +0.39 & -0.19 & -0.59 \\
\hline Kinase Modulator & -0.60 & -0.22 & -0.94 \\
\hline Nuclear Receptor Ligand & -0.17 & -0.04 & -1.02 \\
\hline Protease Inhibitor & -0.01 & -0.15 & -1.27 \\
\hline Enzyme Inhibitor & +0.29 & +0.38 & -0.22 \\
\hline
\end{tabular}

Table 5: ADMET profile of the selected drugs

\begin{tabular}{|c|c|c|c|}
\hline ADMET properties & Levodopa & Esculin & Hinokitol \\
\hline \multicolumn{4}{|l|}{ Absorbtion } \\
\hline Human Intestinal Absorbtion & + & + & + \\
\hline CaCo-2 Permeability & - & - & + \\
\hline P-glycoprotein Substrate & Non-Substrate & Non substrate & Non substrate \\
\hline P-glycoprotein Inhibitor & Non inhibitor & Non inhibitor & Non inhibitor \\
\hline Renal Organic Cation Inhibitor & Non inhibitor & Non inhibitor & Non inhibitor \\
\hline \multicolumn{4}{|l|}{ Distribution, Metabolism } \\
\hline CYP450 2C9 Substrate & Non-Substrate & Non Substrate & Non Substrate \\
\hline CYP450 2D6 Substrate & Non-Substrate & Non Substrate & Non Substrate \\
\hline CYP450 3A4 Substrate & Substrate & Non Substrate & Non Substrate \\
\hline CYP450 1A2 Inhibitor & Inhibitor & Non inhibitor & Inhibitor \\
\hline CYP450 2C9 Inhibitor & Inhibitor & Non inhibitor & Non inhibitor \\
\hline CYP450 2D6 Inhibitor & Inhibitor & Non inhibitor & Non inhibitor \\
\hline CYP450 2C19 Inhibitor & Inhibitor & Non inhibitor & Non inhibitor \\
\hline CYP450 3A4 Inhibitor & Inhibitor & Non inhibitor & Non inhibitor \\
\hline \multirow[t]{2}{*}{ CYP Inhibitory Promiscuity } & Low CYP Inhibitory & Low CYP Inhibitory & Low CYP Inhibitory \\
\hline & Promiscuity & Promiscuity & Promiscuity \\
\hline \multicolumn{4}{|l|}{ Excretion, Toxicity } \\
\hline $\begin{array}{l}\text { Human Etheragogo Related } \\
\text { Gene Inhibition }\end{array}$ & Non-Inhibitor & Non-Inhibitor & Non-Inhibitor \\
\hline AMES Toxicity & AMES Toxic & AMES Toxic & Non-AMES Toxic \\
\hline Carcinogens & Non-Carcinogen & Non-Carcinogen & Non-Carcinogen \\
\hline Fish Toxicity & High & High & High \\
\hline Tetrahymena Pyriformis Toxicity & High & High & High \\
\hline Honey Bee Toxicity & Low & High & High \\
\hline Biodegradation & Readily Biodegradable & Readily Biodegradable & Readily Biodegradable \\
\hline Acute Oral Toxicity & III & III & III \\
\hline \multicolumn{4}{|l|}{ ADMET predicted profile-regression } \\
\hline Aqueous solubility (LogS) & -2.1345 & -4.770 & -4.1536 \\
\hline Caco2 Permeability (LogPapp, cm/s) & -0.6730 & 1.7203 & 0.8364 \\
\hline Rat Acute Toxicity $\left(\mathrm{LD}_{50}, \mathrm{~mol} / \mathrm{kg}\right)$ & 2.3137 & 2.2896 & 2.7972 \\
\hline Fish Toxicity(pLC50, mg/l) & 1.0043 & 1.3261 & -1.4952 \\
\hline Tetrahymena Pyriformis Toxicity (pIGC50, ug/l) & 0.2883 & 0.1235 & 0.6404 \\
\hline
\end{tabular}




\section{DISCUSSION}

Auto Dock uses a semiempirical free energy force field to predict binding free energies of small molecules to macromolecular targets [22]. The force field is based on a comprehensive thermodynamic model that allows incorporation of intramolecular energies into the predicted free energy of binding. The method has been calibrated for a set of 188 diverse protein-ligand complexes of known structure and binding energy, showing a standard error of about 2-3 kcal/Mol in the prediction of binding free energy in cross-validation studies. The docked complex was visualised in AutoDock Tools [23] showing how the ligand interacts with the proteins. At the end of each docking run, AutoDock outputs a result which is the lowest energy conformation of the ligand it found during that run. This conformation is a combination of translation, quaternion and torsion angles and is characterised by intermolecular energy, internal energy and the torsional energy [24]. The first two of these combined forms give the 'docking energy' while the first and third give 'binding energy.' AutoDock also breaks down the total energy into a vdW energy and an electrostatic energy for each atom. The 'best' docking result can be considered to be the conformation with the lowest (docked) energy. The comparative analysis of the two drugs with levodopa helped to find the interacting amino acids in the active sites of all the selected proteins, which are mainly contributing to the neurodegenerative disorder's initiating pathways.

The rapid hydrolysis of acetylcholine (AChE) into acetyl-coA and choline occurs at the termination of the nerve impulse in cholinergic synapses, which is mediated by AChE. Moreover, there is good evidence to provide interrelationship between $\mathrm{TH}$ and brain cholinergic system, with the decreased levels of AChE and the overall deficits to the cholinergic system [25]. TheAS formation plays a divesting role in the progression of neurodegeneration. Besides, in mouse hippocampal neurones, AS has been shown to restrict vesicle diffusion away from synapses, consistent with vesicle clustering inhibition mediated by AS. In rat hippocampal neurons, ASinduced defects in vesicle reclustering following endocytosis was elucidated [26]. Therefore, the inhibition of Tyrosine 156 of the Aposite of AS, in this study paves a new way through which the drug of selection, esculin and hinokitol could act as like of standard levodopa drug. The metabolism of major monoamine neurotransmitters) such as $5-\mathrm{HT}$, norepinephrine and dopamine in the CNS is regulated by a flavoenzyme MAO. It exists in two isoforms namely MAO-A and MAOB. Since time immemorial, many of the human MAO-A inhibitors are used as anti-anxiety as well as antidepressant agents. Whereas, human MAO-B inhibitors are used alone or in combination as anti-Alzheimer's and anti-Parkinsonian drugs [27]. The earlier reports [28] leads to the development of quinazolinone derivatives as anticancer leads by in silico design, in the similar way. In this study, Valine 106 in the case of MOA-B showed better interaction.

Ubiquitin often combines with Parkin as both activator and substrate for modification of Parkin translocation. Phosphorylation of Ser 56 is the reason for both activation and inhibition [11]. This inhibition of Parkin can be another big solution for reducing the progression of neuro-degeneration. In this study, docking studies revealed that the amino acid Thr 240 could be a prospective site of inhibition by esculin as well as hinokitol, in comparison with levodopa.

The molecular physiochemical properties analysis of the drugs revealed that hinokitol was BBB+ showing a positive value for its BBB permeability, assuring it as an affirmative drug for neurodegenerative diseases. Whereas esculin was an uncooperative choice, as its BBB negative and fails to cross BBB. Moreover, the miLogP value of hinokitol was positive, which further confirms its potential to act as an anti-neurodegenerative drug. Furthermore, hinokitol can act as positive GPCR ligand, nuclear receptor as well as protease inhibitor ligands. Thus, this assures that hinokitol could be a better drug of choice for prevention of progression of neurodegenerative diseases like PD. The ADMET studies also support that hinokitol is a good choice to be targeted as an antiParkinsonian drug. However, further, molecular dynamic simulation studies, in vitro as well as in vivo experimentations could clear out the exact function of hinokitol against PD.

\section{CONCLUSION}

The study predicted the potential of hinokitol as a potent antineurodegenerative drug which could target diseases, especially PD, being good inhibitors of AS, MAO-B and Parkin. The drug was predicted to be BBB positive as they could be BBB permeable. The ADMET studies also revealed its ability to act as a high-born drug with minimal toxicity. This in-silico analysis could be taken for further experimentation in in-vitro and in vivo models to reconfirm its potential mechanism of action, so as to utilise the drug as a noble drug for neurodegenerative disorders.

\section{CONFLICT OF INTERESTS}

The authors have no conflict of interest.

\section{REFERENCES}

1. Emma D, Nicholas WW, Hélène PF. Mitophagy and Parkinson's disease: the PINK1-parkin link. Biochim Biophys Acta 2011; 1813:623-33.

2. Falsone SF, Gerd L, Anita K, Andreas JK, Simone K, Roberto C, et al. The neurotransmitter serotonin interrupts $\alpha$-synuclein amyloid maturation. Biochim Biophys Acta 2011;1814:553-61.

3. Leonidas S. a-synuclein in Parkinson's disease. Cold Spring Harbor Perspect Med 2012;4:93-9.

4. Mojtaba G, Elham A, Fatemeh H, Norlinah MI, Behrouz R, Zahurin $\mathrm{M}$, et al. Glycogen synthase kinase3 beta (GSK3 $\beta$ ) signalling: implications for Parkinson's disease. Pharmacol Res 2015;97:16-26.

5. Ericsson AD. Potentiation of the L-Dopa effect in man by the use of catechol-0-methyltransferase inhibitors. J Neurol Sci 1971;14:193-7.

6. Roberts JW, Cora-Locatelli G, Bravi D, Amantea MA, Mouradian MM, Chase TN. Catechol-O-methyltransferase inhibitor tolcapone prolongs levodopa/carbidopa action in Parkinsonian patients. Neurology 1993;43:2685-8.

7. Chrisp P, Mammen, GJ, Sorkin EM. Selegiline-a review of its pharmacology, symptomatic benefits and protective potential in Parkinson's disease. Drugs Aging 1991;1:228-48.

8. Janice HK, Matthias CK, Shirley EP, John SD. Association of a monoamine oxidase B allele with Parkinson's disease. Ann Neurol 1993;3:368-72.

9. Millan MJ, Di Cara BDA, Panayi F, De Groote L, Sicard D, Cistarelli L, et al. Selective blockade of dopamine D versus D receptors enhances frontocortical cholinergic transmission and social memory in rats: a parallel neurochemical and behavioural analysis. J Neurochem 2007;100:1047-61.

10. Peter B, Paola D, Carla B, Johan N, Jansonius, Vladimir NM. Structural insight into Parkinson's disease treatment from drug-inhibited DOPA decarboxylase nature structural biology. Nat Struct Biol 2001;8:963-7.

11. Alicia MP, Richard JY. The roles of PINK1, Parkin and mitochondrial fidelity in parkinson's disease. Neurone 2015;85:257-73.

12. Collier TJ, Lipton J, Daley BF, Palfi, S Chu Y, Sortwell C, et al. Aging-related changes in the nigrostriatal dopamine system and the response to MPTP in nonhuman primates: diminished compensatory mechanisms as a prelude to Parkinsonism. Neurobiol Dis 2007;26:56-65.

13. Shaik JB, Palaka BK, Penumala M, Kotapati KV, Devineni SR, Eadlapalli S, et al. Synthesis, pharmacological assessment, molecular modelling and in silico studies of fused tricyclic coumarin derivatives as a new family of multifunctional antiAlzheimer agents. Eur J Med Chem 2016;107:21932.

14. Casey MC, Richard S, McNally, Brian JC, Tak WM, Jenny PY. DJ-1, a cancer and Parkinson's disease-associated protein, stabilizes the antioxidant transcriptional master regulator Nrf2. PNAS 2006;103:15091-6.

15. Arumugam A, Gunasekaran N, Perumal S. In vitro antioxidant, antidiabetic, cholinesterase and tyrosinase inhibitory potential of fresh juice from Citrus hystrix and C. maxima fruits. Food Sci Hum Wellness 2014;3:16-25.

16. Jayakumar T, Wen-Hsien H, Ting-Lin Y, Jun-Yun L, Yu-Cheng K, Tsorng-Harn F, et al. Hinokitiol, a natural tropolone derivative, offers neuroprotection from a thromboembolic stroke in vivo. Evi 
Based Compland Alternat Med 2013;1-8. Doi:10.1155/ 2013/ 840487.

17. Morris GM, Huey R, Lindstrom W, Sanner MF, Belew RK, Goodsell DS, et al. Auto-Dock 4 and AutoDockTools 4: automated docking with selective receptor flexibility. J Comput Chem 2009;30:2785-91.

18. Mathew B, Suresh J, Anbazghagan S, Paulraj J, Krishnan GK. Heteroaryl chalcones: a mini review about their therapeutic voyage. Biomed Prev Nut 2014;4:451-8.

19. Nayak BV, Yabanoglu SC, Jadav SS, Jagrat M, Sinha BN, Ucar G, et al. Monoamine oxidase inhibitory activity of 3,5-biaryl-4,5dihydro-1Hpyrazole-1-carboxylate derivatives. Eur J Med Chem 2013;69:762-7.

20. Shobana C, Sumathi T, Sujatha L, Sureshkannan S, Kumarasamy P. Molecular docking studies of bacoside-A, an active component of bacopa monniera with DJ1 for antiparkinson drug design. Biomirror 2013;4:67-70.

21. Lipinski CA, Lombardo L, Dominy BW, Feeney PJ. Experimental and computational approaches to estimate solubility and permeability in drug discovery and development settings. Adv Drug Delivery Rev 2001;46:3-26.

22. Huey R, Morris GM, Olson AJ, Goodsell DS. A semiempirical free energy force field with charge-based desolvation. J Comput Chem 2006;28:1145-52.

23. Sanner MF. Python: a programming language for software integration and development. J Mol Graphics Mod 1999;17:57-61.
24. Gopalsamy B, Lawrence X. Molecular docking studies on antiviral drugs for SARS. Int J Adv Res Comput Sci Software Eng 2015;5:75-9.

25. Catherine GV, Caterina C, Dimitra G, Panagiotis G, Marigoula M. Effect of adult-onset hypothyroidism on behavioural parameters and acetylcholinesterase isoforms activity in specific brain regions of male mice. Physiol Behav 2016;164:284-91.

26. Danielle EM, Scott EU, Malcolm JD, Harry I. Dynamic structural flexibility of $\alpha$-synuclein. Neurobiol Dis 2016;88:66-74.

27. Bijo M, Jerad S, Githa EM, Abitha H, Geethu S, Sabreena P. Synthesis, ADME studies, toxicity estimation, and exploration of molecular recognition of thiophene-based chalcones towards monoamine oxidase-A and B. Beni-suef University J Basic Appl Sci 2016. http://dx.doi.org/doi:10.1016/ j.bjbas.2015.06.003

28. Megha S, Amit GN. In silico screening, synthesis and in vitro evaluation of some quinazolinone derivatives as dihydrofolate reductase inhibitors for anticancer activity: Part-I. Int J Pharm Pharm Sci 2014;5:19-9.

\section{How to cite this article}

- Krishnapriya Madhu Varier, Sumathi Thangarajan, Arulvasu Chinnasamy, Gopalsamy Balakrishnan, Renjith Paulose. Comparative analysis of potentiality of Esculin and hinokitol (B-thujaplicin) as anti-parkinsonism drugs: a pilot in silico study. Int J Pharm Pharm Sci 2017;9(1):108-115. 